\title{
Clinically-guided mutation screening of two families with hereditary retinal disease
}

Jian Li ${ }^{1}$, Aierken Yiming ${ }^{1}$, Ping Wang ${ }^{1,2}$

1 Department of Ophthalmology, Kashgar People's Hospital, Kashgar, Xinjiang, China

2 Department of Genetics, Kashgar People's Hospital, Kashgar, Xinjiang, China

Correspondence

Ping Wang

Department of Ophthalmology, Kashgar People's Hospital

Kashgar, Xinjiang, China 843800

Email: Wang.ping@mail.com 


\section{Abstract}

Hereditary retinal disease (HRD) is a series of Mendelian diseases affecting the retina in the eye. The genetic basis of HRD is very complicated, with more than 100 diseasecausing genes being identified. Though NGS has allowed rapid and large-scale mutation screening of Mendelian disease, the cost of NGS still prevents its universal application all over the world, for an accurate molecular diagnosis. Here, by clinical guidance from patient phenotypes, we performed targeted molecular diagnosis by direct Sanger sequencing of the most likely candidate gene in two families diagnosed with HRD. Then we identified two novel protein-truncating variants in the gene CRB1. Our results demonstrated the notion that molecular diagnosis and clinical diagnosis can be mutually supplemented and clinically guided direct sequencing is a cost-effective approach for molecular diagnosis and subsequent genetic counseling. 


\section{Introduction}

Hereditary retinal disease (HRD) is a group of human Mendelian disorders ${ }^{1}$. It includes a number of disease subtypes, including Leber congenital amaurosis, retinitis pigmentosa, cone-rod dystrophy, Stargardt disease, etc.. HRD usually features dysfunction and degeneration of photoreceptors in the retina. Photoreceptor is a highly specialized type of cell in the human body, and its survival and functions are controlled by a number of biological pathways. Therefore, mutations in more than one hundred genes can lead to HRD phenotype ${ }^{2}$. Currently, only $50 \%-70 \%$ of HRD cases can be solved by mutations in known HRD-associated genes. HRD is a relatively more common Mendelian disorder compared with other disease phenotypes, affecting around $1 / 2000$ of the population ${ }^{2}$. This presents a particular need for improved molecular diagnosis.

The power of next-generation sequencing (NGS) has enabled large scale mutation discovery of human Mendelian disorders in recent years. It also speeds up the process of identifying novel disease-causing genes ${ }^{3}$. In the HRD field, the molecular diagnosis of disease cases also benefits a lot from a variety of NGS-based methods, including whole exome sequencing and targeted capture sequencing. Correspondingly, the molecular diagnosis rate of HRD cases has been significantly improved ${ }^{4-13}$, and a series of novel HRD-associated genes have been identified ${ }^{14-33}$. 
However, the cost of NGS approach still prevents its universal application to all the populations affected by HRD, and direct Sanger sequencing is still a cost-effective way to screen for mutations in relatively small genes. Particularly, when a disease phenotype is very likely to be caused by mutations in one or two genes, it is probably not necessary to perform genome-wide mutation screening. Sanger sequencing demonstrates relatively short turn-around time for limited number of reactions, which is particularly useful in targeted analysis.

Here, by clinical evaluation of two disease families of HRD, we narrowed down the disease candidate to one gene, CRB1. Direct Sanger sequencing allowed us to identify two novel protein-truncating variants in this gene, thus showing the strength of Sanger sequencing in some specific scenarios.

\section{Methods and Materials}

\section{Clinical diagnosis}

The HRD disease families were recruited from Kashgar People's Hospital, Kashgar, China for this study. Written informed consent was obtained from participating individuals. Specifically, we performed best visual acuity tests, color vision tests, fundus photography, visual field tests, fundus autofluorescence, and electroretinogram on all the patients for confirming the diagnosis. Family pedigrees were drawn based on interviews. Peripheral blood samples were collected from all available participating 
individuals. Genomic DNA was extracted from blood using the salting-out method ${ }^{34}$. This study adhered to the Declaration of Helsinki. All experimental methods were approved by the ethics committee of Kashgar People's Hospital.

\section{Sanger sequencing and analysis}

Primers were designed to amplify the coding exons of the candidate gene, CRB1. After PCR, the products were run on $1 \%$ agarose gel to confirm the identity. Then the products were sequenced on an $A B I 3730 x I$ machine. The sequence traces were analyzed on FinchTV to search for candidate mutations. GnomAD database was used to annotate the frequency of identified variants ${ }^{35}$. Variants with a population frequency more than $0.5 \%$ was excluded for additional analysis.

\section{Results}

Two families with characteristic HRD phenotypes were included in this study. The Family 1 is a consanguineous family of Uyghur ethnicity. There are two affected individuals in the same generation (Figure 1A). The proband is a 36 -year-old male. He had visual problems at the age of 6 , starting from night blindness. Currently, he has a visual acuity of $20 / 80$ for both eyes and the visual fields are restricted. ERG shows almost diminished responses of rod photoreceptors. Fundus images show bone spicules and very characteristic preserved para-arteriole retinal pigment epithelium (PPRPE) phenotype. This phenotype was shown in RP12 and this locus was later 
identified as the gene $C R B 1^{36 ; 37}$. The other affected individual in this family is a 23year-old female. Similar to her brother, she had night blindness since 7 years old. Now her visual acuity is $20 / 50$ for the left eye and $20 / 40$ for the right eye. ERG test show about $50 \%$ reduction of rod responses. She also has the typical PPRPE phenotypes upon fundus photography. These two patients have five unaffected siblings.

Family 2 is another consanguineous family also with Uyghur ethnicity. Two patients were identified in this family (Figure 1B). The proband is a 30 -year-old female suffering from visual problems since childhood. She has the visual acuity $20 / 50$ for both eyes and ERG responses are absent. She also has a sight nystagmus phenotype. Funduscopy revealed PPRPE phenotypes. She has an 23-year-old affected sister with typical RP phenotypes but without the PPRPE phenotypes.

Due to the very typical phenotype of CRB1-related retinal disease presented in these two families, we decided to use direct Sanger sequencing for mutation screening. For both families, we chose the probands for direct sequencing. After Sanger sequence analysis, we identified two CRB1 protein-truncating mutations in these two families. The proband in Family 1 has a homozygous stopgain mutation in exon 6 (NM_201253, c. $1606 \mathrm{G}>\mathrm{T}$, p.E536*). Similarly, the proband in Family 2 also has a homozygous stopgain mutation in exon 7 (NM_201253, c.2384T>A, p.L795*). Both variants were not reported in HRD patients previously, and they are absent from the gnomAD control database, suggesting they are very rare. The two CRB1 stopgain variants are not 
localized at the last exon, thus they are very likely to be loss-of-function alleles due to nonsense-mediated decay.

Finally, we performed additional Sanger sequencing for the two disease-causing mutations in the additional family members to test the genotype phenotype cosegregation. The genotypes are consistent with the recessive inheritance pattern (Figure 1A and $B$ ).

\section{Discussions}

Sanger sequencing approach was developed several decades ago and it is a robust approach to identify germline variants existing in human DNA. Nowadays, with the availability of NGS, Sanger sequencing is still an indispensable validation method. In this study, we used the Sanger sequencing approach to successfully perform molecular diagnosis on two HRD cases based on their characteristic clinical presentations. These results showed that some typical clinical features of HRD are only caused by mutations in a small set of genes, and this information can help the researchers to narrow down the candidate genes for mutation screening.

CRB1 is a protein localized in the inner segment of the photoreceptor cells ${ }^{38}$. It is the orthologue of the Crumbs protein in the fly. CRB1 also has another paralogue, CRB2, 
also essential for photoreceptor maintenance ${ }^{39}$. However, it seems that CRB2 is indispensable in other tissue, thus mutations in CRB2 lead to early-onset nephrotic syndromes ${ }^{40}$. Researchers have found that CRB1 is essential in maintaining the polarity and integrity of the photoreceptor cells. Mutations in CRB1 also account for a significant proportion of HRD cases, especially for the characteristic PPRPE phenotype.

The mutations of some additional HRD-associated genes can also result in characteristic phenotypes, serving as candidate genes for direct Sanger sequencing when NGS-based approach is not readily available. For example, mutations in $A B C A 4$ can cause typical recessive Stargardt disease phenotypes ${ }^{41}$, and mutations in $\mathrm{CHM}$ can lead to choroideremia ${ }^{42}$. The utilization of the detailed gene-specific clinical outcomes would certainly improve the success rate of targeted Sanger sequencing.

The genetic etiology of $\mathrm{HRD}$ in the population of Uyghur ethnicity is still relatively understudied compared with some other populations, and one recent preliminary study investigated $12 \mathrm{HRD}$ probands using NGS-based targeted exon capture sequencing ${ }^{43}$. Additional mutation screening efforts, especially by NGS-based approach, are needed to investigate the HRD molecular basis of population in this area. This will pave the way for a better genetic counseling and clinical outcome predictions for the HRD patients.

\section{Acknowledgements}


This work was supported by Xinjiang Autonomous District Nature Science Funding (No.31760315). We thank Dr. Lei Wang for helpful suggestions on this project.

\section{References}

1. Francis, P.J. (2006). Genetics of inherited retinal disease. Journal of the Royal Society of Medicine 99, 189-191.

2. Veleri, S., Lazar, C.H., Chang, B., Sieving, P.A., Banin, E., and Swaroop, A. (2015). Biology and therapy of inherited retinal degenerative disease: insights from mouse models. Disease models \& mechanisms 8, 109-129.

3. Yang, Y., Muzny, D.M., Reid, J.G., Bainbridge, M.N., Willis, A., Ward, P.A., Braxton, A., Beuten, J., Xia, F., Niu, Z., et al. (2013). Clinical whole-exome sequencing for the diagnosis of mendelian disorders. The New England journal of medicine 369, $1502-1511$.

4. Li, J., Tang, J., Feng, Y., Xu, M., Chen, R., Zou, X., Sui, R., Chang, E.Y., Lewis, R.A., Zhang, V.W., et al. (2016). Improved Diagnosis of Inherited Retinal Dystrophies by High-Fidelity PCR of ORF15 followed by Next-Generation Sequencing. The Journal of molecular diagnostics : JMD 18, 817-824.

5. Salvo, J., Lyubasyuk, V., Xu, M., Wang, H., Wang, F., Nguyen, D., Wang, K., Luo, H., Wen, C., Shi, C., et al. (2015). Next-generation sequencing and novel variant determination in a cohort of 92 familial exudative vitreoretinopathy patients. Investigative ophthalmology \& visual science 56, 1937-1946. 
6. Zhang, Q., Xu, M., Verriotto, J.D., Li, Y., Wang, H., Gan, L., Lam, B.L., and Chen, R. (2016). Next-generation sequencing-based molecular diagnosis of 35 Hispanic retinitis pigmentosa probands. Scientific reports 6, 32792 .

7. Ventura, M.J., Wheaton, D., Xu, M., Birch, D., Bowne, S.J., Sullivan, L.S., Daiger, S.P., Whitney, A.E., Jones, R.O., Moser, A.B., et al. (2016). Diagnosis of a mild peroxisomal phenotype with next-generation sequencing. Molecular genetics and metabolism reports $9,75-78$.

8. Soens, Z.T., Branch, J., Wu, S., Yuan, Z., Li, Y., Li, H., Wang, K., Xu, M., Rajan, L., Motta, F.L., et al. (2017). Leveraging splice-affecting variant predictors and a minigene validation system to identify Mendelian disease-causing variants among exon-captured variants of uncertain significance. Human mutation 38 , 1521-1533.

9. Sullivan, L.S., Bowne, S.J., Koboldt, D.C., Cadena, E.L., Heckenlively, J.R., Branham, K.E., Wheaton, D.H., Jones, K.D., Ruiz, R.S., Pennesi, M.E., et al. (2017). A Novel Dominant Mutation in SAG, the Arrestin-1 Gene, Is a Common Cause of Retinitis Pigmentosa in Hispanic Families in the Southwestern United States. Investigative ophthalmology \& visual science 58, 2774-2784.

10. Daiger, S.P., Sullivan, L.S., Bowne, S.J., Birch, D.G., Heckenlively, J.R., Pierce, E.A., and Weinstock, G.M. (2010). Targeted high-throughput DNA sequencing for gene discovery in retinitis pigmentosa. Advances in experimental medicine and biology 664, 325-331.

11. Carrigan, M., Duignan, E., Malone, C.P., Stephenson, K., Saad, T., McDermott, C., Green, A., Keegan, D., Humphries, P., Kenna, P.F., et al. (2016). Panel-Based 
Population Next-Generation Sequencing for Inherited Retinal Degenerations. Scientific reports 6, 33248 .

12. Weisschuh, N., Mayer, A.K., Strom, T.M., Kohl, S., Glockle, N., Schubach, M., Andreasson, S., Bernd, A., Birch, D.G., Hamel, C.P., et al. (2016). Mutation Detection in Patients with Retinal Dystrophies Using Targeted Next Generation Sequencing. PloS one 11, e0145951.

13. Zhao, L., Wang, F., Wang, H., Li, Y., Alexander, S., Wang, K., Willoughby, C.E., Zaneveld, J.E., Jiang, L., Soens, Z.T., et al. (2015). Next-generation sequencingbased molecular diagnosis of 82 retinitis pigmentosa probands from Northern Ireland. Human genetics 134, 217-230.

14. Xu, M., Yamada, T., Sun, Z., Eblimit, A., Lopez, I., Wang, F., Manya, H., Xu, S., Zhao, L., Li, Y., et al. (2016). Mutations in POMGNT1 cause non-syndromic retinitis pigmentosa. Human molecular genetics 25, 1479-1488.

15. Arno, G., Carss, K.J., Hull, S., Zihni, C., Robson, A.G., Fiorentino, A., Hardcastle, A.J., Holder, G.E., Cheetham, M.E., Plagnol, V., et al. (2017). Biallelic Mutation of ARHGEF18, Involved in the Determination of Epithelial Apicobasal Polarity, Causes Adult-Onset Retinal Degeneration. American journal of human genetics $100,334-342$.

16. Arno, G., Agrawal, S.A., Eblimit, A., Bellingham, J., Xu, M., Wang, F., Chakarova, C., Parfitt, D.A., Lane, A., Burgoyne, T., et al. (2016). Mutations in REEP6 Cause Autosomal-Recessive Retinitis Pigmentosa. American journal of human genetics $99,1305-1315$. 
17. Xu, M., Gelowani, V., Eblimit, A., Wang, F., Young, M.P., Sawyer, B.L., Zhao, L., Jenkins, G., Creel, D.J., Wang, K., et al. (2015). ATF6 Is Mutated in Early Onset Photoreceptor Degeneration With Macular Involvement. Investigative ophthalmology \& visual science 56, 3889-3895.

18. Fu, Q., Xu, M., Chen, X., Sheng, X., Yuan, Z., Liu, Y., Li, H., Sun, Z., Li, H., Yang, L., et al. (2017). CEP78 is mutated in a distinct type of Usher syndrome. Journal of medical genetics $54,190-195$.

19. Xu, M., Eblimit, A., Wang, J., Li, J., Wang, F., Zhao, L., Wang, X., Xiao, N., Li, Y., Wong, L.J., et al. (2016). ADIPOR1 Is Mutated in Syndromic Retinitis Pigmentosa. Human mutation 37, 246-249.

20. Xu, M., Xie, Y.A., Abouzeid, H., Gordon, C.T., Fiorentino, A., Sun, Z., Lehman, A., Osman, I.S., Dharmat, R., Riveiro-Alvarez, R., et al. (2017). Mutations in the Spliceosome Component CWC27 Cause Retinal Degeneration with or without Additional Developmental Anomalies. American journal of human genetics 100, $592-604$.

21. Saksens, N.T., Krebs, M.P., Schoenmaker-Koller, F.E., Hicks, W., Yu, M., Shi, L., Rowe, L., Collin, G.B., Charette, J.R., Letteboer, S.J., et al. (2016). Mutations in CTNNA1 cause butterfly-shaped pigment dystrophy and perturbed retinal pigment epithelium integrity. Nature genetics 48, 144-151.

22. Vincent, A., Audo, I., Tavares, E., Maynes, J.T., Tumber, A., Wright, T., Li, S., Michiels, C., Condroyer, C., MacDonald, H., et al. (2016). Biallelic Mutations in GNB3 Cause a Unique Form of Autosomal-Recessive Congenital Stationary Night Blindness. American journal of human genetics 98, 1011-1019. 
23. Coppieters, F., Ascari, G., Dannhausen, K., Nikopoulos, K., Peelman, F., Karlstetter, M., Xu, M., Brachet, C., Meunier, I., Tsilimbaris, M.K., et al. (2016). Isolated and Syndromic Retinal Dystrophy Caused by Biallelic Mutations in RCBTB1, a Gene Implicated in Ubiquitination. American journal of human genetics 99, 470-480.

24. Yuan, Z., Li, B., Xu, M., Chang, E.Y., Li, H., Yang, L., Wu, S., Soens, Z.T., Li, Y., Wong, L.C., et al. (2017). The phenotypic variability of HK1-associated retinal dystrophy. Scientific reports $7,7051$.

25. Zhao, L., Chen, Y., Bajaj, A.O., Eblimit, A., Xu, M., Soens, Z.T., Wang, F., Ge, Z., Jung, S.Y., He, F., et al. (2016). Integrative subcellular proteomic analysis allows accurate prediction of human disease-causing genes. Genome research 26, 660669.

26. Agrawal, S.A., Burgoyne, T., Eblimit, A., Bellingham, J., Parfitt, D.A., Lane, A., Nichols, R., Asomugha, C., Hayes, M.J., Munro, P.M., et al. (2017). REEP6 deficiency leads to retinal degeneration through disruption of ER homeostasis and protein trafficking. Human molecular genetics 26, 2667-2677.

27. Wang, F., Li, H., Xu, M., Li, H., Zhao, L., Yang, L., Zaneveld, J.E., Wang, K., Li, Y., Sui, R., et al. (2014). A homozygous missense mutation in NEUROD1 is associated with nonsyndromic autosomal recessive retinitis pigmentosa. Investigative ophthalmology \& visual science 56, 150-155.

28. Wang, F., Wang, Y., Zhang, B., Zhao, L., Lyubasyuk, V., Wang, K., Xu, M., Li, Y., Wu, F., Wen, C., et al. (2014). A missense mutation in HK1 leads to autosomal dominant retinitis pigmentosa. Investigative ophthalmology \& visual science 55 , 7159-7164. 
29. Xu, M., Yang, L., Wang, F., Li, H., Wang, X., Wang, W., Ge, Z., Wang, K., Zhao, L., Li, H., et al. (2015). Mutations in human IFT140 cause non-syndromic retinal degeneration. Human genetics 134, 1069-1078.

30. Soens, Z.T., Li, Y., Zhao, L., Eblimit, A., Dharmat, R., Li, Y., Chen, Y., Naqeeb, M., Fajardo, N., Lopez, I., et al. (2016). Hypomorphic mutations identified in the candidate Leber congenital amaurosis gene CLUAP1. Genetics in medicine : official journal of the American College of Medical Genetics 18, 1044-1051.

31. Bujakowska, K.M., Zhang, Q., Siemiatkowska, A.M., Liu, Q., Place, E., Falk, M.J., Consugar, M., Lancelot, M.E., Antonio, A., Lonjou, C., et al. (2015). Mutations in IFT172 cause isolated retinal degeneration and Bardet-Biedl syndrome. Human molecular genetics 24, 230-242.

32. El-Asrag, M.E., Sergouniotis, P.I., McKibbin, M., Plagnol, V., Sheridan, E., Waseem, N., Abdelhamed, Z., McKeefry, D., Van Schil, K., Poulter, J.A., et al. (2015). Biallelic mutations in the autophagy regulator DRAM2 cause retinal dystrophy with early macular involvement. American journal of human genetics 96, 948954.

33. Yang, P., Chiang, P.W., Weleber, R.G., and Pennesi, M.E. (2015). Autosomal Dominant Retinal Dystrophy With Electronegative Waveform Associated With a Novel RAX2 Mutation. JAMA ophthalmology 133, 653-661.

34. Miller, S.A., Dykes, D.D., and Polesky, H.F. (1988). A simple salting out procedure for extracting DNA from human nucleated cells. Nucleic acids research 16, 1215. 35. Lek, M., Karczewski, K.J., Minikel, E.V., Samocha, K.E., Banks, E., Fennell, T., O'Donnell-Luria, A.H., Ware, J.S., Hill, A.J., Cummings, B.B., et al. (2016). 
Analysis of protein-coding genetic variation in 60,706 humans. Nature 536, $285-$ 291.

36. Heckenlively, J.R. (1982). Preserved para-arteriole retinal pigment epithelium (PPRPE) in retinitis pigmentosa. The British journal of ophthalmology 66, 26-30.

37. den Hollander, A.I., ten Brink, J.B., de Kok, Y.J., van Soest, S., van den Born, L.I., van Driel, M.A., van de Pol, D.J., Payne, A.M., Bhattacharya, S.S., Kellner, U., et al. (1999). Mutations in a human homologue of Drosophila crumbs cause retinitis pigmentosa (RP12). Nature genetics 23, 217-221.

38. Quinn, P.M., Pellissier, L.P., and Wijnholds, J. (2017). The CRB1 Complex:

Following the Trail of Crumbs to a Feasible Gene Therapy Strategy. Frontiers in neuroscience 11, 175 .

39. Alves, C.H., Pellissier, L.P., Vos, R.M., Garcia Garrido, M., Sothilingam, V., Seide, C., Beck, S.C., Klooster, J., Furukawa, T., Flannery, J.G., et al. (2014). Targeted ablation of Crb2 in photoreceptor cells induces retinitis pigmentosa. Human molecular genetics 23, 3384-3401.

40. Ebarasi, L., Ashraf, S., Bierzynska, A., Gee, H.Y., McCarthy, H.J., Lovric, S., Sadowski, C.E., Pabst, W., Vega-Warner, V., Fang, H., et al. (2015). Defects of CRB2 cause steroid-resistant nephrotic syndrome. American journal of human genetics 96, 153-161.

41. Koenekoop, R.K. (2003). The gene for Stargardt disease, ABCA4, is a major retinal gene: a mini-review. Ophthalmic genetics 24, 75-80.

42. van den Hurk, J.A., van de Pol, T.J., Molloy, C.M., Brunsmann, F., Ruther, K., Zrenner, E., Pinckers, A.J., Pawlowitzki, I.H., Bleeker-Wagemakers, E.M., 
Wieringa, B., et al. (1992). Detection and characterization of point mutations in the choroideremia candidate gene by PCR-SSCP analysis and direct DNA sequencing. American journal of human genetics 50, 1195-1202.

43. Tajiguli, A., Xu, M., Fu, Q., Yiming, R., Wang, K., Li, Y., Eblimit, A., Sui, R., Chen, R., and Aisa, H.A. (2016). Next-generation sequencing-based molecular diagnosis of 12 inherited retinal disease probands of Uyghur ethnicity. Scientific reports 6,21384 . 


\section{Figure Legends}

Figure $1 \mathrm{~A}$. The pedigrees and genotypes of the two families in this study.

Figure 1B. The location of the two novel CRB1 variants identified in this study.

A

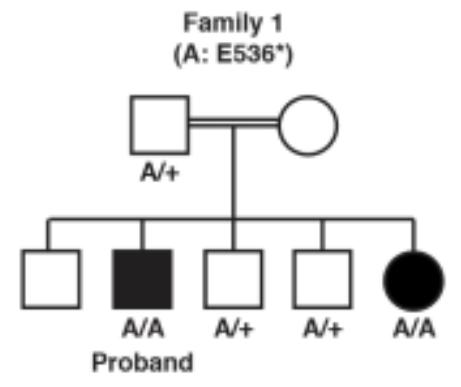

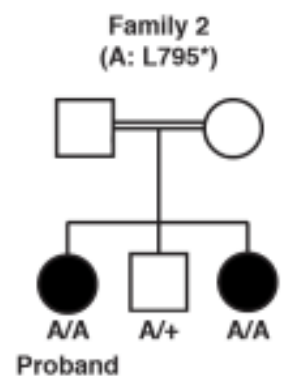

B

Human
CRB1

